\title{
Cellulose-specific Type B carbohydrate binding modules: understanding oligomeric and non-crystalline substrate recognition mechanisms
}

\author{
Abhishek A. Kognole and Christina M. Payne*
}

\begin{abstract}
Background: Effective enzymatic degradation of crystalline polysaccharides requires a synergistic cocktail of hydrolytic enzymes tailored to the wide-ranging degree of substrate crystallinity. To accomplish this type of targeted carbohydrate recognition, nature produces multi-modular enzymes, having at least one catalytic domain appended to one or more carbohydrate binding modules (CBMs). The Type B CBM categorization encompasses several families (i.e., protein folds) of CBMs that are generally thought to selectively bind oligomeric polysaccharides; however, a subset of cellulose-specific CBM families (17 and 28) appear to bind non-crystalline cellulose more tightly than oligomers and in a manner that discriminates between surface topology.
\end{abstract}

Results: To provide insight into this unexplained phenomenon, we investigated the molecular-level origins of oligomeric and non-crystalline carbohydrate recognition in cellulose-specific Type B CBMs using molecular dynamics (MD) simulation and free energy calculations. Examining two CBMs from three different families (4, 17, and 28), we describe how protein-ligand dynamics contribute to observed variations in binding affinity of oligomers within the same CBM family. Comparisons across the three CBM families identified factors leading to modified functionality prohibiting competitive binding, despite similarity in sequence and specificity. Using free energy perturbation with Hamiltonian replica exchange MD, we also examined the hypothesis that the open topology of the binding grooves in families 17 and 28 necessitates tight binding of an oligomer, while the more confined family 4 binding groove does not require the same degree of tight binding. Finally, we elucidated the mechanisms of non-crystalline carbohydrate recognition by modeling CBMs complexed with a partially decrystallized cellulose substrate. Molecular simulation provided structural and dynamic data for direct comparison to oligomeric modes of carbohydrate recognition, and umbrella sampling MD was used to determine ligand binding free energy. Comparing both protein-carbohydrate interactions and ligand binding free energies, which were in good agreement with experimental values, we confirmed the hypothesis that family 17 and 28 CBMs bind non-crystalline cellulose and oligomers with different affinities (i.e., high and low).

Conclusions: Our study provides an unprecedented level of insight into the complex solid and soluble carbohydrate substrate recognition mechanisms of Type B CBMs, the findings of which hold considerable promise for enhancing lignocellulosic biomass conversion technology and development of plant cell wall probes.

Keywords: Amorphous cellulose, $\beta$-Sandwich fold, Carbohydrate-aromatic stacking, Free energy perturbation, Multimodular glycoside hydrolases

*Correspondence: christy.payne@uky.edu

Department of Chemical and Materials Engineering, University

of Kentucky, 177 F Paul Anderson Tower, Lexington, KY 40506, USA 


\section{Background}

Enzymatic hydrolysis of lignocellulosic biomass into fermentable sugars remains a technologically challenging step in the cost-effective production of secondgeneration biofuels [1]; both enzyme stability and rate of hydrolytic turnover are targets for biotechnological improvement. Substrate composition and crystallinity, as well as efficient substrate recognition by enzymes, are a few of the important factors that determine the yield and rate of enzymatic hydrolysis [2]. Microorganisms often accomplish carbohydrate hydrolysis through the secretion of multi-modular enzymes, wherein carbohydrate binding modules (CBMs) are appended to globular catalytic domains via a linker peptide. CBMs play a valuable role in hydrolysis and recognition processes, as they are responsible for maintaining proximity of the enzyme to the substrate and targeting specific regions of crystallinity [3-5]. These functions serve to enhance catalytic turnover under substrate-limited conditions [6-8]. The ability to target distinct morphological regions of carbohydrate substrates is perhaps the most interesting, yet least understood, function of CBMs and offers great potential in extending the industrial application of CBMs beyond lignocellulosic biomass conversion [9-13]. For example, CBMs show promise as biotechnological tools in applications as wide ranging as affinity chromatography, targeting of functional molecules to cellulosic substrates, cell immobilization technology, enzyme engineering, and fiber modification $[4,5,8]$. As such, there is a critical need to develop understanding of the molecular-level carbohydrate recognition process of CBMs.

CBMs are structurally diverse proteins, binding with many different types of carbohydrate polymorphs and morphologies. To capture this diversity categorically, CBMs have been divided into both families and types based on protein sequence and functional similarity, respectively $[3,14]$. Currently, this nomenclature defines function as the ability to target particular substrate crystallinities, as CBMs appear to bind either crystalline or non-crystalline/amorphous and oligomeric substrates. Type A CBMs are specific for crystalline substrates and exhibit a complementary planar binding site lined with aromatic residues $[15,16]$. Type B and C CBMs are only subtly different from each other, with both types generally binding oligosaccharides and non-crystalline/amorphous substrates in clefts or grooves. Type B CBMs are capable of binding at any point along the length of the substrate, and Type C CBMs are limited to the end of the oligomer. The underlying protein features enabling this distinction are difficult to define.

The most common protein fold among Type B CBMs is the $\beta$-sandwich fold, the proteins of which uniquely recognize not only different kinds of carbohydrates but also varying degrees of polymerization, from the smallest of oligosaccharides to amorphous substrates [3]. This suggests the $\beta$-sandwich fold is a versatile architecture that allows relatively minor variations in sequence and, accordingly, chemical properties of the binding cleft/ groove to determine carbohydrate binding specificity. Moreover, despite similar substrate specificities and, in some cases, similar measured affinities, some Type B CBMs appear to uncompetitively discriminate between binding sites on variable crystallinity surfaces [16, 17]. Attempts to experimentally characterize non-crystalline/ amorphous cellulose have revealed few details of specific structural properties, only that it is cellulose with a decreasing degree of polymerization and crystallinity index [18]. Non-crystalline/amorphous cellulose derived from pretreatment of native crystalline cellulose could be composed of anything from variable-length polysaccharide chains to only partially decrystallized substrate. Thus, the ability to recognize both soluble oligomers and non-crystalline/amorphous cellulose is a key aspect of Type B CBM functionality.

Cellulose-specific Type B CBMs, including those from families 4, 17, and 28, each with the $\beta$-sandwich fold (Fig. 1), have been shown to bind both soluble cello-oligomers and non-crystalline cellulose [6-8, 19-23]. Additionally, adsorption isotherms suggest families 17 and 28 individually recognize 'high' and 'low' affinity binding sites on representative non-crystalline cellulose substrates [13]. These studies also reveal that family 17 and 28 Type B CBMs exhibit higher affinities towards noncrystalline cellulose than toward oligomeric substrates [13, 24]. Cellulomonas fimi CBM4-1 and CBM4-2 (CfCBM4-1 and CfCBM4-2, respectively) also appear to bind cello-oligomers bi-directionally, with the reducing end of the pyranose ring at either end of the cleft; there is positive, but limited, evidence of this phenomenon being common among $\beta$-sandwich CBMs $[11,25]$. Collectively, the data imply that these Type B CBMs are discriminating between the various available binding sites on the non-crystalline carbohydrate surface, but there is not necessarily a directional preference within the binding site.

To gain a molecular-level understanding of how these three families of Type B CBMs discriminate between binding soluble oligomeric and non-crystalline/amorphous substrates, we implemented a computational approach to describe the differences in binding behavior and affinities within and among the CBM families. From molecular dynamics (MD) simulations, we explored the role of binding site architecture. Free energy perturbation with Hamiltonian replica exchange MD (FEP/ $\lambda$-REMD) and umbrella sampling $M D$ was used to examine bidirectional ligand binding ability and apparent binding 

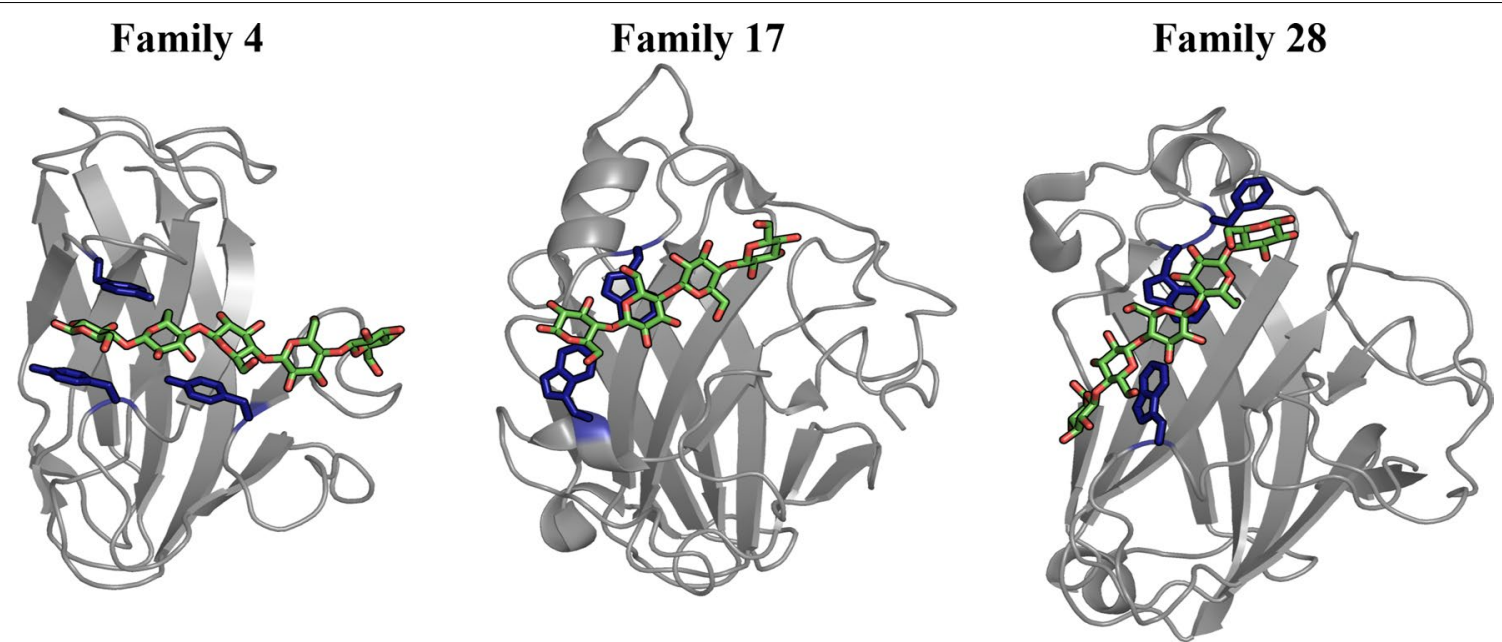

Fig. 1 CBMs (cartoon) from families 4, 17, and 28 with bound cello-oligomers (green and red sticks). Binding site aromatic residues are shown in a dark blue stick representation. The structures, Cellulomonas fimi CBM4-1, Clostridium cellulovorans CBM17, and Clostridium josui CBM28, were obtained from crystal structures with PDB IDs $1 \mathrm{GU} 3,1 \mathrm{~J} 84$, and $3 \mathrm{ACl}$, respectively. After structural alignment of the $\beta$-sandwich proteins, the family 4 and 17 CBM cello-oligomer is bound in the same direction, with the reducing end toward the left of the figure, whereas the family 28 CBM's cello-oligomer is oriented in the opposite direction

modes in non-crystalline substrate recognition. At each step of our study, we compared the computational results with available experimental data to assess the validity of our observations and to translate observations to practice. Two representative CBMs from each of the three CBM families, 4,17 , and 28 , were selected to gain an understanding of the variations in protein-carbohydrate binding within and across the families. The selected representatives were Cellulomonas fimi CBM4-1 and CBM4-2 (CfCBM4-1 and CfCBM4-2), Clostridium cellulovorans CBM17 (CcCBM17), Bacillus sp. 1139 CBM17 and CBM28 (BspCBM17 and BspCBM28), and Clostridium josui CBM28 (CjCBM28). The representatives were selected on the basis that they are characterized as cellulose-specific Type B CBMs and are shown to have affinity for both oligomeric and non-crystalline cellulose. CBMs with available structural data were preferred to be able to convincingly apply computational techniques. Briefly, the protein-carbohydrate systems were modeled in the following configurations (Fig. 2): (A) CBMs with the cellooligosaccharide bound in the orientation observed in the crystallographic structure, (B) CBMs with the oligosaccharide bound in the opposite direction of the structural orientation (i.e., with the reducing end of the sugar longitudinally rotated to the opposite end of the groove), and (C) CBMs bound with a partially decrystallized cellulose microfibril, representative of non-crystalline cellulose, in both the structural and reverse orientations. Additionally, each of the CBM representatives was modeled without a bound ligand for comparison, totaling 16 unique molecular models (Additional file 1: Table S1). The "Methods" section contains additional details on model construction to aid in understanding the "Results and discussion" section.

\section{Results and discussion}

Role of binding site architecture in substrate recognition

The three CBM families, 4, 17, and 28, share the same $\beta$-sandwich protein fold but exhibit key differences in binding site architectures/platforms. As they all belong to the Type B classification, the binding site generally conforms to either a cleft or groove capable of accommodating a single glycan chain. However, structural examination reveals the family 4 CBMs exhibit much deeper binding clefts relative to the more open grooves of family 17 and $28 \mathrm{CBMs}$, which we expect plays a critical role in substrate recognition mechanisms. Both CfCBM4-1 and CfCBM4-2 display aromatic residues lining the cleft and whose hydrophobic surfaces face each other to sandwich the substrate pyranose rings between them (Fig. 3). The oligomeric substrate is enveloped in a 4-5 $\AA$-deep cleft with its pyranose ring perpendicular to the CBM surface [26]. Family 17 and 28 CBM binding grooves also display aromatic residues, although they are positioned side-byside with their hydrophobic surfaces exposed to the solvent. Additionally, these aromatic residues are not exactly aligned in parallel planes, as in Type A CBMs, but, rather, comprise a shallow 1-2 ̊̊ groove with a 'twisted' polysaccharide-binding platform [24, 27].

The significance of individual hydrophobic aromatic residues and polar residues in both family 17 and 28 CBMs has been examined in prior experimental studies 


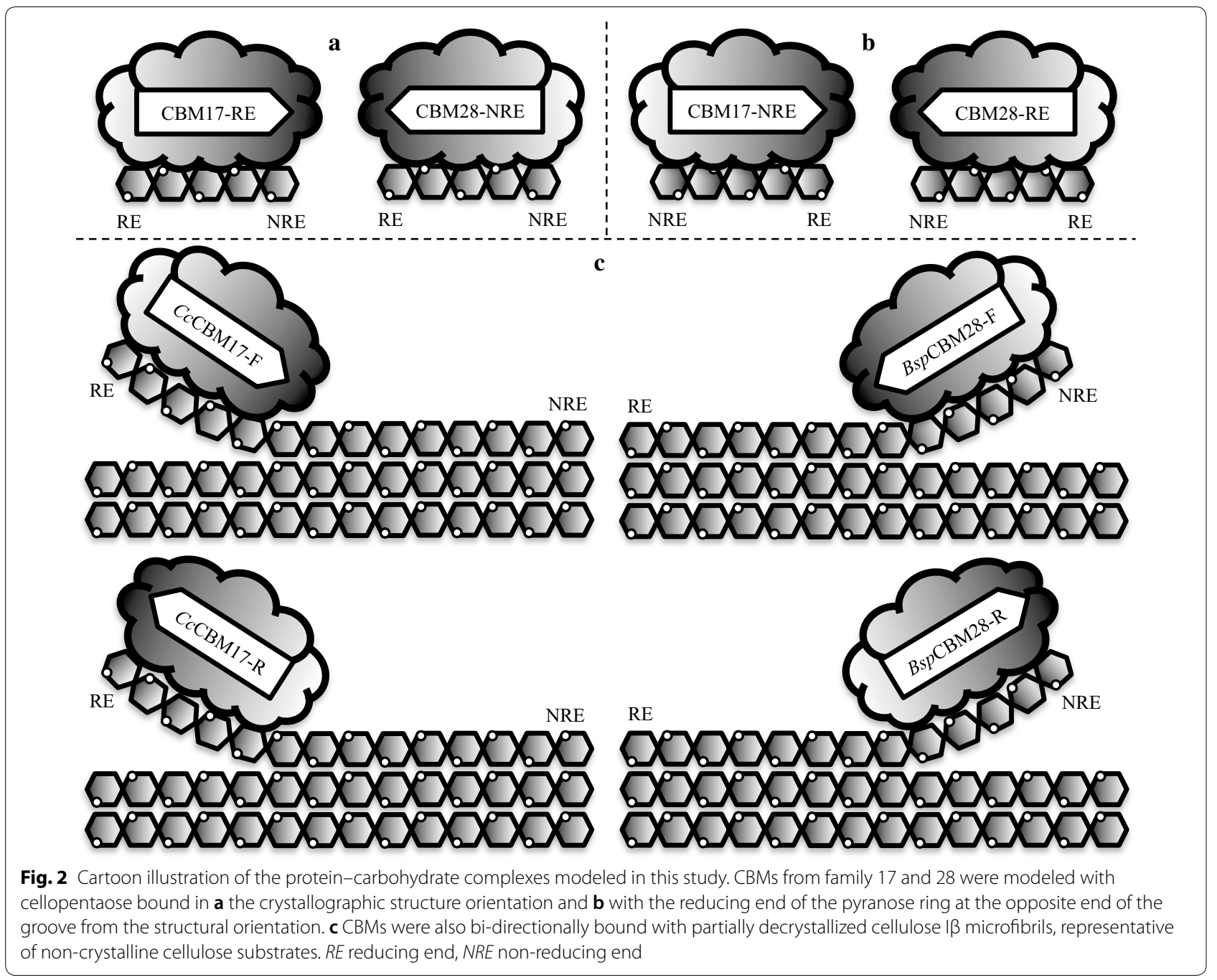

[24, 28, 29]; however, binding affinity studies suggest that, despite structural and sequence similarity, thermodynamic binding signatures are not always consistent within members of the same family [21, 27]. We have previously discussed the similarities and differences within the two family 4 CBMs for ligand binding dynamics and thermodynamic preference [25]. Here, we focus on comparing and contrasting oligomeric ligand binding modes and affinity across the two different binding platforms of CBM4s and CBM17 and 28s, 'sandwich' and 'twisted', respectively, as well as within and across the three Type $B$ CBM families.

CfCBM4-1-RE and CfCBM4-2-RE (sandwich platforms), and CcCBM17-RE and CjCBM28-NRE (twisted platforms) are compared here, as experimental binding affinities and structures have been determined for each. Reported affinities for cellopentaose for each of the four CBMs, as well as structurally similar family members, are given in Table 1. Note that measurement techniques and experimental conditions vary by study making direct comparison challenging. For the same four CBM-cellopentaose systems, we calculated binding affinity using FEP/ $\lambda$-REMD method under conditions similar to experiment, at $300 \mathrm{~K}$ and $\mathrm{pH}$ 7.0. The orientation of the ligands relative to the CBM binding cleft reflected the crystallographic configuration (Fig. 2a). We found that CfCBM4-1 and CfCBM4-2 exhibited affinities for cellopentaose of $-4.5 \pm 1.3 \mathrm{kcal} / \mathrm{mol}[25]$ and $-5.4 \pm 1.4 \mathrm{kcal} /$ mol. CcCBM17-RE and CjCBM28-NRE exhibited affinities for cellopentaose of $-6.9 \pm 0.9 \mathrm{kcal} / \mathrm{mol}$ and $-6.3 \pm 0.7 \mathrm{kcal} / \mathrm{mol}$, respectively. Detailed distribution of free energy components, including charge, dispersion, van der Waals, and restraining contributions (Table S2), and illustration of calculation convergence has been provided in Additional file 1: Fig. S3. While the trend is weak, the experimental and calculated affinities, combined, suggest moderately tighter cellopentaose binding may occur in some 'twisted platform' CBMs (i.e., families 


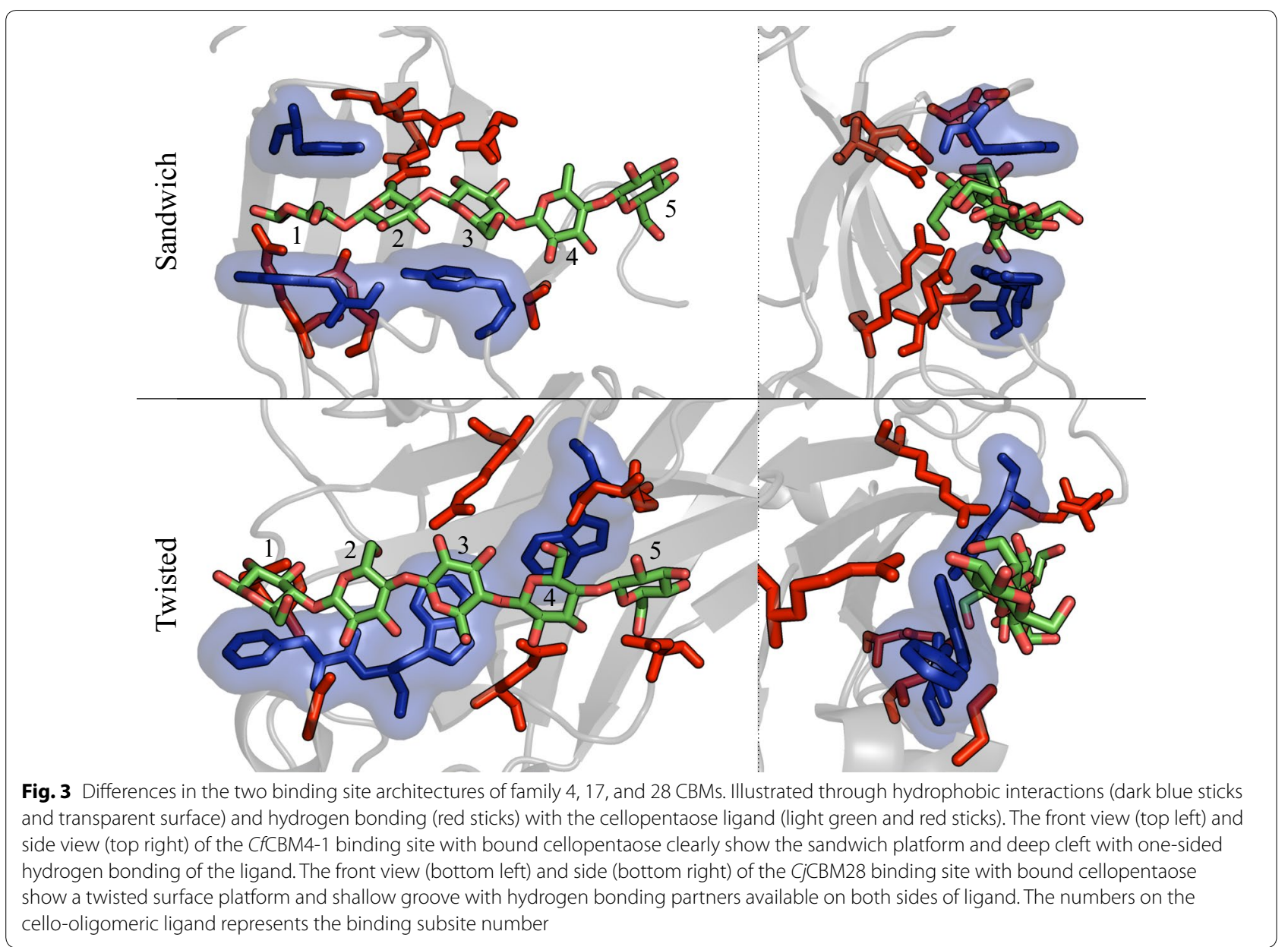

17 and 28). These subtle thermodynamic preferences of different platforms are among the factors that play a role in building the recognition mechanisms targeted towards specific substrates, as we discuss below. Later in this study, we address our hypothesis that moderately tighter binding in the twisted platform is an evolutionary feature of family 17 and 28 CBMs that allows them to preferably recognize non-crystalline cellulose over cello-oligomers.

\section{MD simulations further differentiate binding platforms}

MD simulations provide additional insight into the binding free energy calculations, revealing that $\mathrm{CcCBM} 17-$ $\mathrm{RE}$ and CjCBM28-NRE form more stable non-covalent interactions with the cellopentaose ligand than either family 4 CBM. From the 250-ns MD trajectories, we calculated the root mean square fluctuation (RMSF) of the ligand on a per-binding-subsite basis (Fig. 4); error was estimated by block averaging over 2.5 ns blocks. This value describes how much a given pyranose ring fluctuates from its average position over the course of a simulation. Collectively, as well as in nearly every binding site, the pyranose rings within the CfCBM4-1 and $C f C B M 4-2$ binding cleft fluctuate more than that of either CcCBM17-RE or CjCBM28-NRE, indicating the latter two ligands form more stable protein-carbohydrate contacts. Moreover, the lower RMSF combined with the marginally higher binding affinity in the twisted platforms suggests that the unfavorable entropic penalty is compensated by enthalpic contributions, particularly hydrogen bonding.

There are three aromatic residues in the binding sites of the CBM4s and CBM28s, while CBM17s display only two, so the contribution to ligand binding from hydrophobic stacking interactions is not platform-dependent, varying by family. Rather, hydrogen bonding interactions appear to be a key determinant in affinity differences between the two binding site architectures. The average number of hydrogen bonds formed between a given pyranose ring with the side chains of the surrounding protein was determined using VMD; detailed analysis of hydrogen bonding over the course of the MD simulations identified the primary hydrogen bonding partners in all 
Table 1 Binding affinities of a given family 4, 17, or 28 CBM for cellopentaose

\begin{tabular}{|c|c|c|c|c|c|c|}
\hline References & CBM & Method & Temperature (K) & $\mathrm{pH}$ & Buffer conditions & $\Delta G^{\circ}(\mathrm{kcal} / \mathrm{mol})$ \\
\hline Tomme et al. [23] & CfCBM4-1 & ITC & 308 & 7.0 & Pure water & $-5.23 \pm 0.91$ \\
\hline Tomme et al. [23] & CfCBM4-1 & $\mathrm{ITC}$ & 308 & 7.0 & 50 mM potassium phosphate & $-6.09 \pm 0.64$ \\
\hline Tomme et al. [23] & CfCBM4-1 & $\mathrm{ITC}$ & 308 & 7.0 & $\begin{array}{l}50 \mathrm{mM} \text { potassium phosphate } \\
1.0 \mathrm{M} \mathrm{NaCl}\end{array}$ & $-6.21 \pm 0.57$ \\
\hline Brun et al. [58] & CfCBM4-2 & $\mathrm{ITC}$ & 308 & 7.0 & $\begin{array}{l}50 \mathrm{mM} \text { potassium phosphate } \\
1.0 \mathrm{M} \mathrm{NaCl}\end{array}$ & $-5.80 \pm 0.005$ \\
\hline Boraston et al. [22] & CcCBM17 & Fluorescence & 298 & 7.5 & 25 mM Tris- $\mathrm{HCl}$ & -6.72 \\
\hline Boraston et al. [22] & CcCBM17 & Fluorescence & 298 & 7.5 & $\begin{array}{l}25 \mathrm{mM} \text { Tris- } \mathrm{HCl} \\
0.5 \mathrm{M} \mathrm{NaCl}\end{array}$ & -6.93 \\
\hline Boraston et al. [22] & CcCBM17 & Fluorescence & 298 & 7.5 & $\begin{array}{l}25 \mathrm{mM} \text { Tris- } \mathrm{HCl} \\
1.0 \mathrm{M} \mathrm{NaCl}\end{array}$ & -7.05 \\
\hline Boraston et al. [22] & CcCBM17 & Fluorescence & 298 & 7.5 & $\begin{array}{l}25 \mathrm{mM} \text { Tris- } \mathrm{HCl} \\
2.0 \mathrm{M} \mathrm{NaCl}\end{array}$ & -7.24 \\
\hline Notenboom et al. [24] & CcCBM17 & ITC & 298 & 7.0 & 50 mM potassium phosphate & $-5.80 \pm 0.03$ \\
\hline Boraston et al. [21] & Bsp CBM28 & UV & 298 & 7.0 & 25 mM Tris- $\mathrm{HCl}$ & $-5.81 \pm 0.14$ \\
\hline Boraston et al. [21] & Bsp CBM28 & ITC & 298 & 7.0 & 50 mM potassium phosphate & $-5.93 \pm 0.003$ \\
\hline Araki et al. [29] & CjCBM17 & ITC & 293 & 7.0 & 100 mM potassium phosphate & $-7.7 \pm 0.3$ \\
\hline Araki et al. [29] & CjCBM28 & ITC & 293 & 7.0 & 100 mM potassium phosphate & $-7.7 \pm 0.6$ \\
\hline
\end{tabular}

The CBMs listed here include: Cellulomonas fimi CBM4-1 and CBM4-2 (CfCBM4-1 and CfCBM4-2), Clostridium cellulovorans CBM17 (CcCBM17), Bacillus sp. 1139 CBM28 (BspCBM28), and Clostridium josui CBM28 (CjCBM28). The methods for obtaining binding affinities in the referenced study are listed as either isothermal titration calorimetry (ITC), fluorescence emission scans, or ultraviolet (UV) difference spectra. Reported experimental conditions are as given

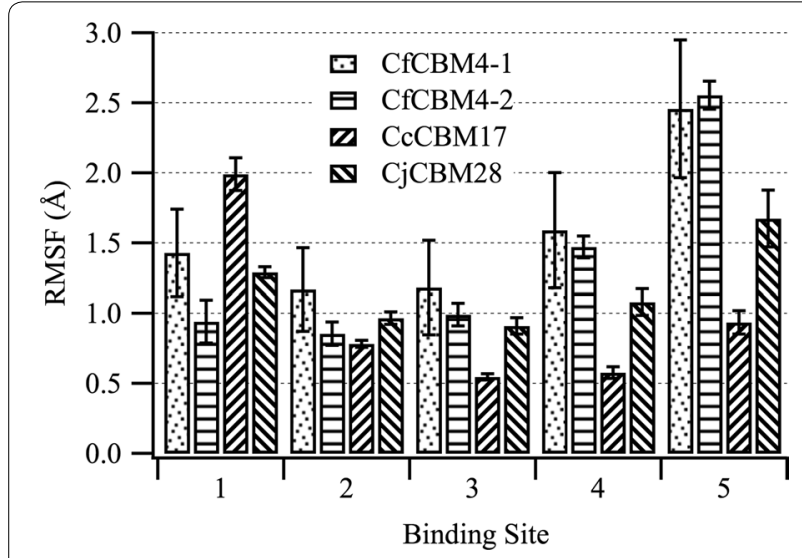

Fig. 4 Root mean square fluctuation (RMSF) of cellopentaose. RMSF of the cellopentaose ligand from its average position in the clefts/ grooves of representatives from family 4, 17, and 28 CBMs obtained from 250-ns MD simulation on a per-binding-subsite basis. Error was calculated from block averaging with block sizes of $2.5 \mathrm{~ns}$

the CBM-oligomer interactions. The average number of hydrogen bonds formed per binding site was calculated from the 250-ns MD trajectories, where a hydrogen bond was defined as two polar atoms having a donor-acceptor distance of $<3.0 \AA$ and a $20^{\circ}$ cutoff angle. Table 2 shows the hydrogen bonding pairs from the calculations along with percent occupancy of each pair, where occupancy refers to the percent of the simulation during which the hydrogen bond was formed. While the CBMs with the same binding site architecture exhibit comparable hydrogen bonding, the total number of hydrogen bonds formed with the twisted platform was almost $100 \%$ higher than that of the sandwich platform. Total percent occupancy of $100 \%$ indicates that at any given time of simulation there is, on average, at least one hydrogen bond between the ligand and protein. Along the twisted platform, there are one or more additional hydrogen bonding partners, accounting for an additional $1-2 \mathrm{kcal} / \mathrm{mol}$ of binding free energy for the whole binding site [30, 31]. CcCBM17-RE and CjCBM28-NRE form more hydrogen bonds with cellopentaose than either CfCBM4-1 and CfCBM4-2, fitting with the conjecture that the loss of conformational entropy in ligand binding is compensated by enthalpic contributions to free energy. This difference in hydrogen bonding can be justified by analysis of the positioning of partner amino acid residues along the binding site. In the sandwich platform, where the ligand is approximately perpendicular to the protein surface, primary and secondary hydroxyl groups of only one edge of the cellopentaose chain contact the CBM and the other edge is exposed to solvent (Fig. 3). In contrast, the cellopentaose bound in the twisted platform hydrogen bonds with partners on both sides of the groove. In CBM4s, there are relatively few hydrogen bonding partners available at binding subsite 5, but in the case of CcCBM17-RE and CjCBM28-NRE, each binding subsite exhibits at least one residue capable of hydrogen bonding. 
Table 2 Percent occupancy of each hydrogen bond formed between the pyranose ring at each binding site and the surrounding protein residue over the 250 -ns simulation

\begin{tabular}{|c|c|c|c|c|c|c|}
\hline & \multicolumn{3}{|l|}{ CfCBM4-1 } & \multicolumn{3}{|l|}{ CfCBM4-2 } \\
\hline & Donor & Acceptor & Occupancy (\%) & Donor & Acceptor & Occupancy (\%) \\
\hline \multirow[t]{15}{*}{ Sandwich platform } & ARG75-Side & BGC1-Side & 26.86 & ARG81-Side & BGC1-Side & 43.83 \\
\hline & BGC3-Side & ASN81-Side & 25.70 & BGC2-Side & GLN128-Side & 40.02 \\
\hline & BGC4-Side & ALA18-Main & 25.14 & HSE132-Side & BGC2-Side & 26.11 \\
\hline & BGC2-Side & TYR43-Main & 16.61 & BGC4-Side & LEU24-Main & 7.83 \\
\hline & BGC2-Side & GLN124-Side & 15.37 & BGC4-Side & SER23-Side & 6.96 \\
\hline & GLN128-Side & BGC1-Side & 6.54 & BGC3-Side & SER23-Side & 5.24 \\
\hline & GLY82-Main & BGC2-Side & 5.11 & ASN56-Side & BGC3-Side & 3.98 \\
\hline & GLN124-Side & BGC3-Side & 2.23 & SER23-Side & BGC3-Side & 3.27 \\
\hline & BGC4-Side & ASN50-Side & 1.90 & SER23-Side & BGC4-Side & 2.86 \\
\hline & ASN50-Side & BGC3-Side & 1.57 & GLN128-Side & BGC2-Side & 1.47 \\
\hline & BGC2-Side & ASN81-Main & 1.43 & - & - & - \\
\hline & BGC3-Side & GLN124-Side & 1.11 & - & - & - \\
\hline & GLN124-Side & BGC2-Side & 1.04 & & & \\
\hline & \multicolumn{3}{|l|}{ CCCBM17-RE } & \multicolumn{3}{|l|}{ CjCBM28-NRE } \\
\hline & Donor & Acceptor & Occupancy (\%) & Donor & Acceptor & Occupancy (\%) \\
\hline \multirow[t]{12}{*}{ Twisted platform } & BGC3-Side & ASP54-Side & 63.20 & ARG83-Side & BGC3-Side & 65.98 \\
\hline & BGC4-Side & GLN129-Side & 59.43 & ARG178-Side & BGC1-Side & 54.69 \\
\hline & ARG92-Side & BGC2-Side & 37.35 & BGC4-Side & GLN131-Side & 45.93 \\
\hline & BGC2-Side & ASP54-Side & 27.80 & BGC5-Side & ASP76-Side & 26.08 \\
\hline & GLN129-Side & BGC3-Side & 20.06 & BGC2-Side & GLY127-Main & 23.77 \\
\hline & ASN185-Side & BGC4-Side & 15.67 & GLY77-Main & BGC5-Side & 7.54 \\
\hline & ASN137-Side & BGC1-Side & 14.64 & BGC5-Side & ASP135-Side & 3.14 \\
\hline & ASN52-Side & BGC3-Side & 9.25 & BGC4-Side & ASP76-Side & 2.68 \\
\hline & THR184-Side & BGC5-Side & 2.64 & GLN131-Side & BGC4-Side & 1.67 \\
\hline & ARG92-Side & BGC1-Side & 2.15 & TRP129-Side & BGC4-Side & 1.57 \\
\hline & BGC5-Side & THR184-Main & 1.54 & TRP78-Main & BGC5-Side & 1.12 \\
\hline & BGC1-Side & ASN137-Side & 1.08 & - & - & - \\
\hline
\end{tabular}

Data are shown in decreasing order of occupancy. Pairs with occupancy lower than $1 \%$ are not shown. BGC is an acronym for $\beta$-D-glucose. A hydrogen bond was defined as two polar atoms having a donor-acceptor distance of $<3.0 \AA$ and a $20^{\circ}$ cutoff angle

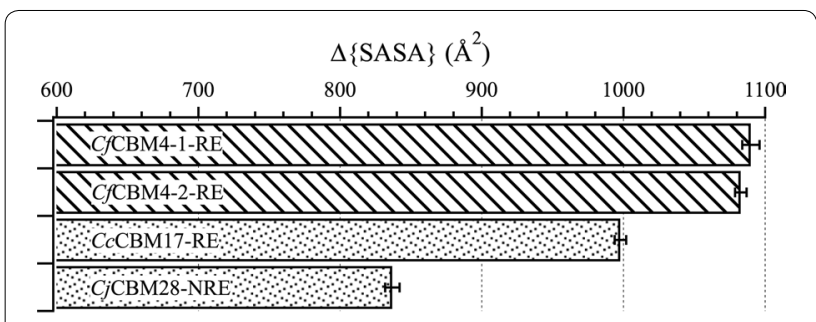

$\Delta\{$ SASA $\}=\operatorname{Avg}\{$ SASA $($ Prot $)\}+\operatorname{Avg}\{$ SASA(Lig) $\}-\operatorname{Avg}\{$ SASA(Prot+Lig) $\}$

Fig. 5 Average change in solvent accessible surface area ( $\triangle S A S A$ ) upon ligand binding. $\triangle S A S A$ is calculated using VMD over the 250-ns MD simulation trajectories of each CBM-cellopentaose system to compare the difference between sandwich (lined pattern) and twisted (dotted pattern) platforms. The error bars represent the standard deviation of the mean
Average change in solvent accessible surface area (SASA) upon ligand binding (Fig. 5) reveals that the sandwich platform buried more solvent exposed surface area upon binding than the twisted platform, although the latter was more solvent exposed initially. The average change in SASA was calculated over 2500 frames of MD simulation, taking the difference between summation of average SASA of apo CBMs and average SASA of solvated cellopentaose, and average SASA of respective CBM-cellopentaose complexes (Fig. 5). The mean change in SASA is lower for twisted platform CBMs than sandwich platform CBMs, with less of a change in SASA observed for CjCBM28-NRE than CcCBM17-RE. The extra aromatic residue (Phe128) in the CjCBM28 binding groove, being the most obvious difference within the twisted platforms of family 17 and $28 \mathrm{CBMs}$, appears to contribute to this difference, but it also suggests that 
having an aromatic residue may not always contribute to higher change in SASA as compared to sandwich platform CBMs that have three aromatic residues. Solventexposed residues along the twisted platforms do not appear to retain ordered water molecules proximal to the aromatic side chains when there is no bound ligand; upon ligand binding, additional water molecules were retained at the protein-carbohydrate interface, as hydroxyl groups of cello-oligosaccharides enable solvent reorganization [27]. The larger change in SASA for sandwich platform CBMs also reflects a conformational change upon ligand binding. As observed in the MD simulations, the deep cleft of the two family 4 CBMs narrows over time as it sandwiches the cello-oligomer and excludes water from the hydrophobic core of the protein. The twisted platform, on the other hand, does not appear to implement this sandwiching mechanism and may, rather, prefer sliding along the polysaccharide chain more freely.

Overall, MD simulation results, particularly through the observed hydrogen bonding patterns, suggest that the difference in cleft architecture (i.e., twisted vs. sandwich) contributes to differences in cellopentaose binding and, likely, protein-carbohydrate recognition mechanisms. It is tempting to suggest variations in molecular-level behavior, such as these, are a result of evolutionary necessity, where each binding site architecture is uniquely suited for targeting regional substrate features $[13,24]$.

\section{Extended binding sites of the twisted platform}

To further differentiate oligomeric recognition mechanisms between CcCBM17 and CjCBM28, we compared ligand binding dynamics at each binding subsite (Fig. 6). Despite the apparent similarity in binding site architecture, the two CBMs feature cello-oligomers bound in opposite directions in their crystal structures (i.e., with the reducing end oriented at a different end of the groove when structurally aligned) [24, 27]. To enable comparison, the binding subsites of the two CBMs were structurally aligned, and a letter-based subsite nomenclature was invoked based on the two common solvent-exposed Trp residues (Fig. 6). The RMSF and hydrogen bonding evaluations reported above follow the numbered binding subsite nomenclature from crystal structure publications, as a cumulative comparison across the platforms. Alignment and renaming binding subsites (A to F), as previously implemented by Tsukimoto et al. [27], reveals that four common binding subsites $(\mathrm{B}, \mathrm{C}, \mathrm{D}$, and $\mathrm{E})$ are occupied by cellopentaose in CcCBM17-RE (B to F) and CjCBM28-NRE (A to E).

The average total interaction energy of protein with the cellopentaose ligand was determined from the 250ns trajectory on a per-binding-subsite basis. The interaction energy distribution was very similar for CBM17 and
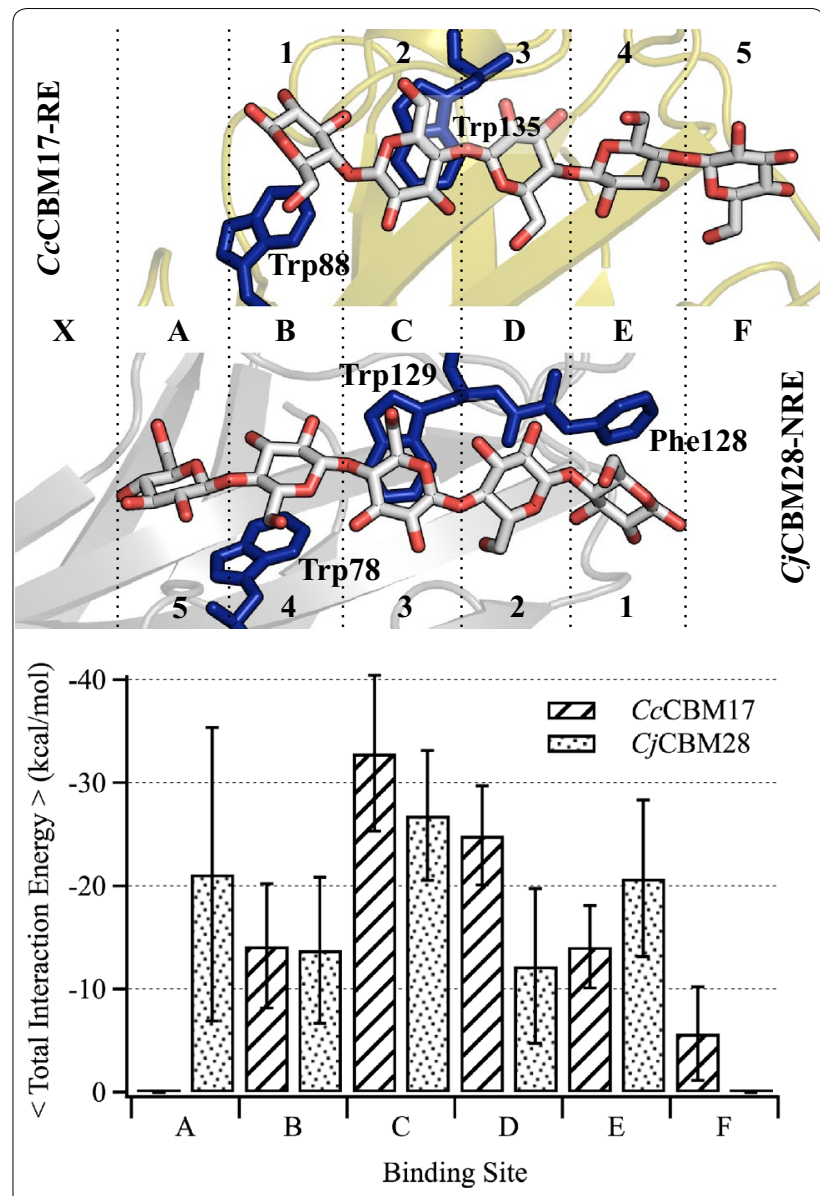

Fig. 6 Alignment and comparison of the twisted platform binding sites. CCCBM17-RE and CjCBM28-NRE aligned with respect to the common pair of Trp residues (dark blue sticks) (top). The common naming of binding subsites used in this study (letters) is given between the alignment of the binding sites, and the original nomenclature (numbers) is given above and below the cartoon representations in the top panel. Average total interaction energy of the pyranose rings with the surrounding amino acid residues, on a per-subsite-basis, of CCCBM17-RE and CjCBM28-NRE calculated from the 250-ns trajectory (bottom). Error bars represent 1 standard deviation

CBM28 in the binding subsites $\mathrm{B}$ and $\mathrm{C}$ that reside along the hydrophobic face of a pair of Trp residues common to both CBMs (Fig. 6). Differences arise in the binding subsites as the extra aromatic residue in family $28 \mathrm{CBMs}$ (Phe128 in CjCBM28 and Tyr118 in BspCBM28) that can provide hydrophobic stacking interaction at binding site E. Nevertheless, we observe little difference at subsites $\mathrm{D}$ and $\mathrm{E}$ in the total interaction energy calculation that accounts for both van der Waals and electrostatic interactions. CBM17s and CBM28s are reported to bind oligomers as long as cellohexaose [21, 24], and it is apparent that, for CBM17s, the sixth subsite would be A, while 
for CBM28s, the sixth sugar can be accommodated in either $\mathrm{F}$ or X. As CcCBM17 and CjCBM28 are known to bind non-crystalline substrates as well, it is possible secondary binding subsites exist for chains even longer than cellohexaose. Accordingly, we docked cellohexaose with CjCBM28 in two orientations, occupying subsites A to $\mathrm{F}$ and $\mathrm{X}$ to $\mathrm{E}$, and conducted 100-ns MD simulations; these simulations showed that both subsite $\mathrm{X}$ and F functionally interact with the ligand, although X had a higher interaction than subsite $F$ (Additional file 1: Fig. S4). Extended binding sites may play a critical role in the recognition of non-crystalline substrates.

\section{Bi-directional ligand binding extends to family 17 and 28 CBMs}

In a previous study, we found that family 4 CBMs showed no thermodynamic preference towards a given longitudinal orientation of cello-oligomers (i.e., the oligomers can bind 'bi-directionally' with the reducing end of the chain at either end of the cleft); moreover, structural comparison of all 29 available (as of the publication date, June 2015) ligand-bound CBM structures exhibiting a $\beta$-sandwich fold revealed bi-directional binding occurs in many other $\beta$-sandwich CBM families [25]. We hypothesized bi-directional binding may be a feature $\beta$-sandwich CBMs developed as an evolutionary advantage, given that bi-directional binding could increase the probability of binding events up to twofold.

Here, we investigate the feasibility and mechanisms of the bi-directional binding phenomenon in family 17 and 28 CBMs. Although there are architectural differences in the binding sites of family 4 and family 17 and $28 \mathrm{CBMs}$, the approximate symmetry of cello-oligomeric ligands and redundancy of available hydrogen bonding partners in the cleft are the determining factors in bi-directionality, which is transferable over the architectures. To consider bi-directional binding within the twisted platform CBMs, we investigated the binding dynamics of four CBMs from families 17 and 28 (CcCBM17, BspCBM17, $B s p$ CBM28, and CjCBM28) with the cellopentaose ligand bound in both possible orientations in the binding grooves.

In all eight simulation cases, the bound cellopentaose ligand maintained continuous interaction with the CBM binding surface over the entire 250-ns simulations, indicating that the binding sites of these family 17 and 28 CBMs can generally accommodate cello-oligomers bidirectionally. The RMSF of the ligand in the binding site provides a quantitative measure of stability of the interactions (Fig. 7), and while all four CBMs can accommodate the ligand bi-directionally, not all of them exhibit fully stabilized protein-ligand interactions. CcCBM17$\mathrm{RE}, B s p \mathrm{CBM} 28-\mathrm{NRE}$, and CjCBM28-NRE bind the
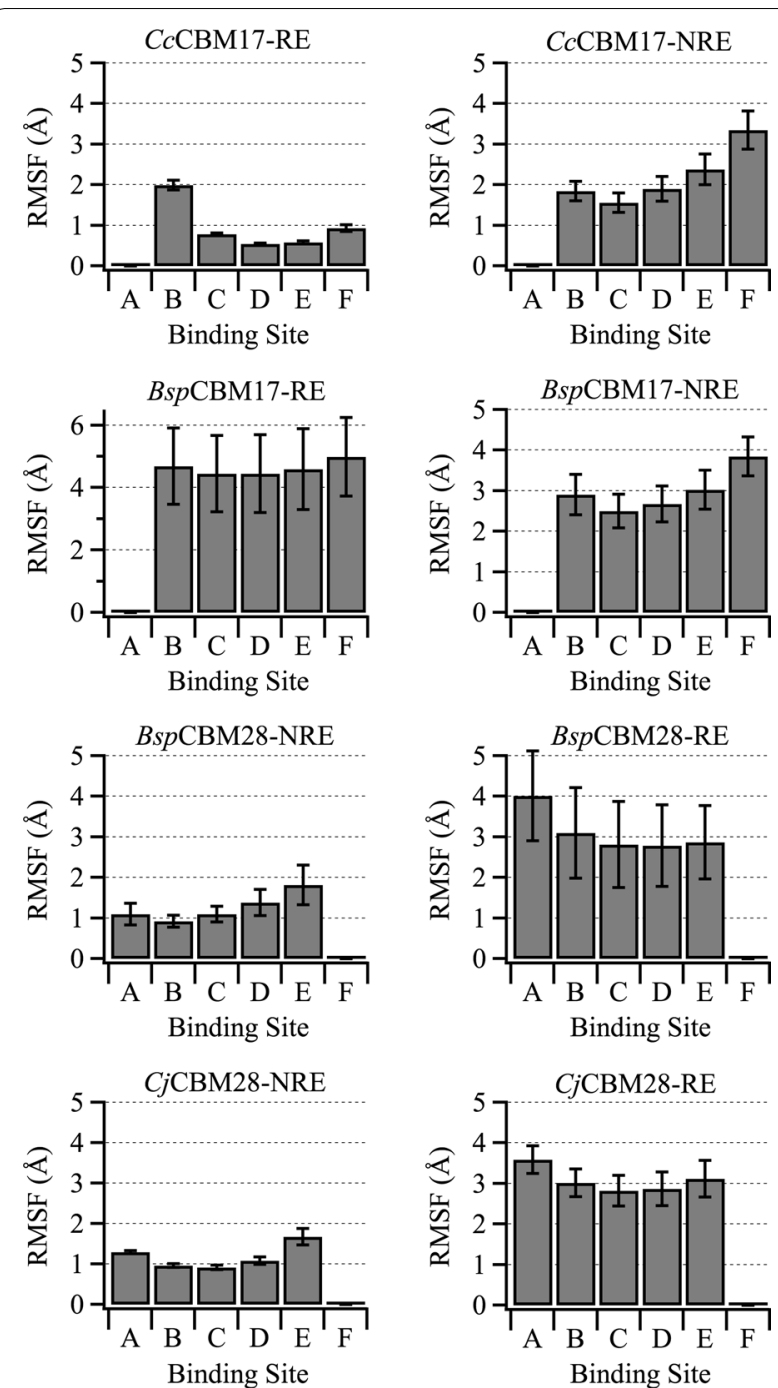

Fig. 7 Root mean square fluctuation (RMSF) of the ligand. RMSF of the cellopentaose ligand from its average position over 250-ns trajectories. The RMSF was calculated on a per-binding-subsite basis for all eight family 17 and 28 CBM-cellopentaose systems. The error bars represent the standard deviation of block averaged RMSFs with block sizes of 2.5 ns each

cello-oligomer with relatively little fluctuation about the average $(\sim 1 \AA)$. In the remaining five cases, though the cellopentaose ligands maintain contact with the CBM binding grooves, we observed sliding of the cellopentaose ligand along the binding site, which is reflected in the increased RMSF. We previously observed cellopentaose sliding within the CBM4 binding sites, however, the oligomers moved only a single subsite in either direction to rearrange the primary hydroxyl groups within the groove, as a result of the purposeful perturbation of ligand orientation [25]. The sliding observed in BspCBM17-RE, by two subsites or a cellobiose unit, maintains the primary 
and secondary hydroxyl group positions within a given subsite, which is suggestive of a functional mechanism rather than merely alleviation of steric hindrance. A cluster of snapshots (every $2.5 \mathrm{~ns}$ ) from each simulation has been provided in Additional file 1 illustrating this phenomenon (Additional file 1: Fig. S5). In the case of BspCBM17-NRE, between 85 and 100 ns, the cellopentaose slides by one subsite, but an accompanying flip around the longitudinal axis maintains the original hydroxyl group orientation within the groove. Again, these results suggest the family 17 and 28 CBMs feature extended binding sites capable of binding cellohexaose or longer oligomers. Based on the alignment in Fig. 6, cellopentaose occupied subsites $\mathrm{X}$ and $\mathrm{A}$ in $B s p \mathrm{CBM} 17-\mathrm{RE}$ and subsite A in BspCBM17-NRE (Additional file 1: Fig. S5).

These simulations support the hypothesis that the twisted platform of cellulose-specific CBMs also displays the characteristics of a binding site capable of bidirectionally binding cello-oligosaccharides. There exists a possibility that the uni-directional binding of cello-oligomers in these CBM crystal structures may have been favored by given conditions of crystallization, while in solution, these Type B CBMs have no thermodynamic preference for an orientation. However, experimental validation of this bi-directional binding phenomenon is desirable.

\section{Differentiation of high and low affinity binding sites on non-crystalline cellulose}

Structural characterizations of many carbohydrateactive enzymes focus strictly on the interactions occurring in the carbohydrate binding site or catalytic active sites, while protein surface residues or secondary binding sites may be just as important to functionality [32]. Type B CBMs are reported to bind both cello-oligomers and non-crystalline/amorphous cellulose, covering a broad range of polymeric structural diversity and suggesting recognition processes may involve interactions beyond the primary binding site. Interestingly, CBMs from families 17 and 28 appear to bind non-crystalline cellulose with high and low binding affinities, as determined from isothermal titration calorimetry (ITC) data, and the two families do not compete with each other for carbohydrate binding sites $[13,33]$. We further explore both the concept of bi-directional binding and the high/low binding affinity phenomena of family 17 and 28 CBMs on noncrystalline cellulose by modeling representative Type B CBMs bound with a model non-crystalline substrate in multiple orientations. At the nanoscale, we propose a partially decrystallized cellulose $\mathrm{I} \beta$ microfibril sufficiently represents the interaction of a CBM with noncrystalline cellulose, which retains a significant degree of crystallinity. Additionally, given our above insights into family 17 and $28 \mathrm{CBM}$ members (i.e., that there is relatively little difference in binding dynamics of oligomers between members of the same family), we modeled only four representative CBM-microfibril systems: one CBM from each family attached to the decrystallized chain, or 'whisker,' in two possible orientations, forward and reverse (Fig. 2c). Details of this have been provided in the methods section below.

To explore the primary modes of Type B carbohydrate recognition with respect to non-crystalline cellulose, we used fully atomistic MD simulations based on previously validated cellulase-cellulose models [34]. All atomic interactions were unbiased except for the lower layer of the cellulose microfibril, which was harmonically restrained to prevent excessive fraying and further decrystallization. In all four cases, CcCBM17-F, CcCBM17-R, BspCBM28-F, and BspCBM28-R (Fig. 2c), the CBM-non-crystalline cellulose complexes stabilized in a global minimum state within the 100-ns MD simulations, illustrated by the rapid plateau in the protein backbone RMSD over time (Additional file 1: Fig. S6). Throughout the simulation, most CBMs bound all five pyranose moieties of the whisker along the twisted binding sites in the fully decrystallized state; in the case of BspCBM28-F, the fifth pyranose ring closest to the cellulose surface partially re-annealed into the microfibril, which is not unexpected [34]. Comparing RMSF of the CBM backbone when bound to either an oligomer or non-crystalline cellulose reveals that ligand binding stabilized the protein (lower RMSF); unbound CBM RMSFs exhibited larger fluctuations near binding site residues in both CBMs. Only BspCBM28-RE, bound with the rotated cellopentaose, showed large protein backbone fluctuations (Additional file 1: Fig. S7), resulting from ligand movement along the binding groove (Fig. 7) (Additional file 1: Fig. S7).

Comparing these four simulations and the oligomerbound simulations above, we identified molecular-level factors contributing to substrate recognition in each family with respect to variation in substrate and orientation. The interaction energy of each CBM residue with the substrate was determined by averaging the calculation over trajectories, for each of the CBM-substrate systems (Fig. 8). For both family 17 and 28 CBMs, the average interaction of a given CBM residue with the substrate was independent of direction of cellopentaose ligand in the binding site, which is, again, consistent with bi-directional ligand binding. The hydrophobic-stacking aromatic residues and hydrogen bonding partners of the CBMcellopentaose systems, as discussed above, produce substantial favorable interaction energies $(<-5 \mathrm{kcal} / \mathrm{mol})$. These same residue-substrate interactions exist when the $\mathrm{CBM}$ is bound with non-crystalline cellulose. However, 


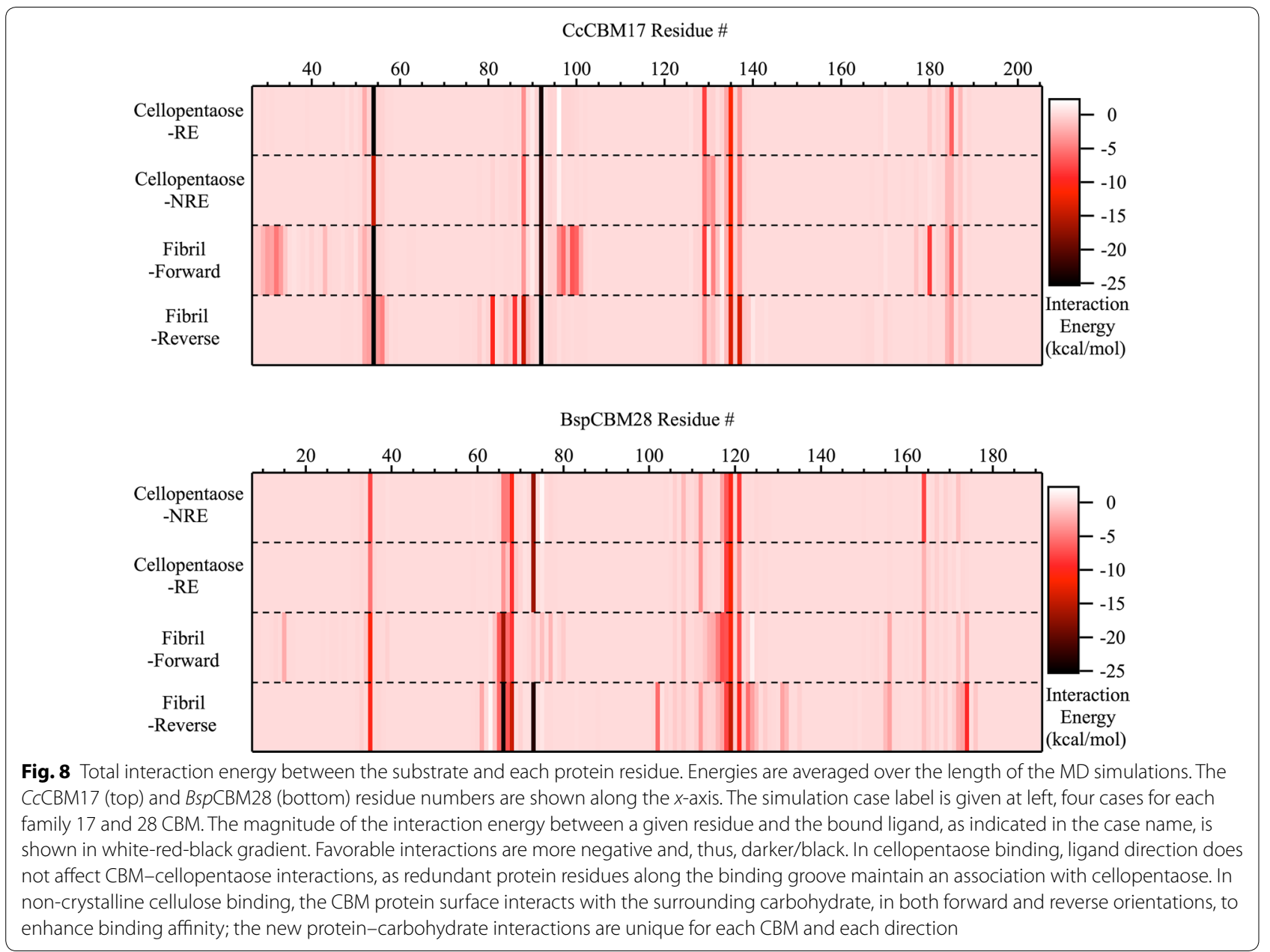

additional protein residues along the CBM surface also appear to be involved in binding non-crystalline cellulose (Fig. 8), as revealed from the rather significant new interactions formed in regions where the CBM-cellopentaose systems produce no such interactions.

While it is clear that protein surface residues play an auxiliary role in non-crystalline cellulose binding, each $\mathrm{CBM}$ and orientation relative to the cellulose surface results in a unique set of protein-substrate interactions to amplify non-crystalline cellulose binding affinity over oligomeric affinity. In the case of CcCBM17-F, two long peptide loops exterior to the binding groove, residues 30-35 and 95-106, interacted with cellulose as a result of their proximity to the cellulose surface in this 'forward' orientation. Most residues in these loops are polar residues, including Pro31, Lys32, Asp33, Asp96, Gln100, Ser101, Asn103, and Tyr105, and served to anchor the CBM over the microfibril through additional hydrogen bonding. In the case of CcCBM17-R, Asp81, Asn86, and Asn137 produced new, large electrostatic interactions between the CBM and substrate. Also, aromatic residues like Trp88 produced more favorable interaction energies in the reverse orientation, while interacting loops in the forward orientation played no role at all. Similarly, for BspCBM28-F, the family 28 CBM lost hydrogen bond interactions between the ligand and Arg73 in the binding groove (subsite 3) and Gln112 (subsite 4); however, new hydrogen bond interactions with residues in loop 65-68 were formed. The BspCBM28-R orientation exhibited more consistent interaction patterns, with no loss of affinity contributors and formation of additional favorable interactions between cellulose and residues in loops 66-68 and 115-130. Ultimately, it seems each orientation of a given CBM relative to the cellulose surface produces a specific set of substrate interactions that enhance non-crystalline cellulose binding relative to binding of oligomers.

To thermodynamically characterize the effects of orientation and substrate crystallinity on family 17 and 28 binding, we calculated binding affinities from the potential of mean force (PMF), or work required, to separate the CBMs from the non-crystalline cellulose substrate. 

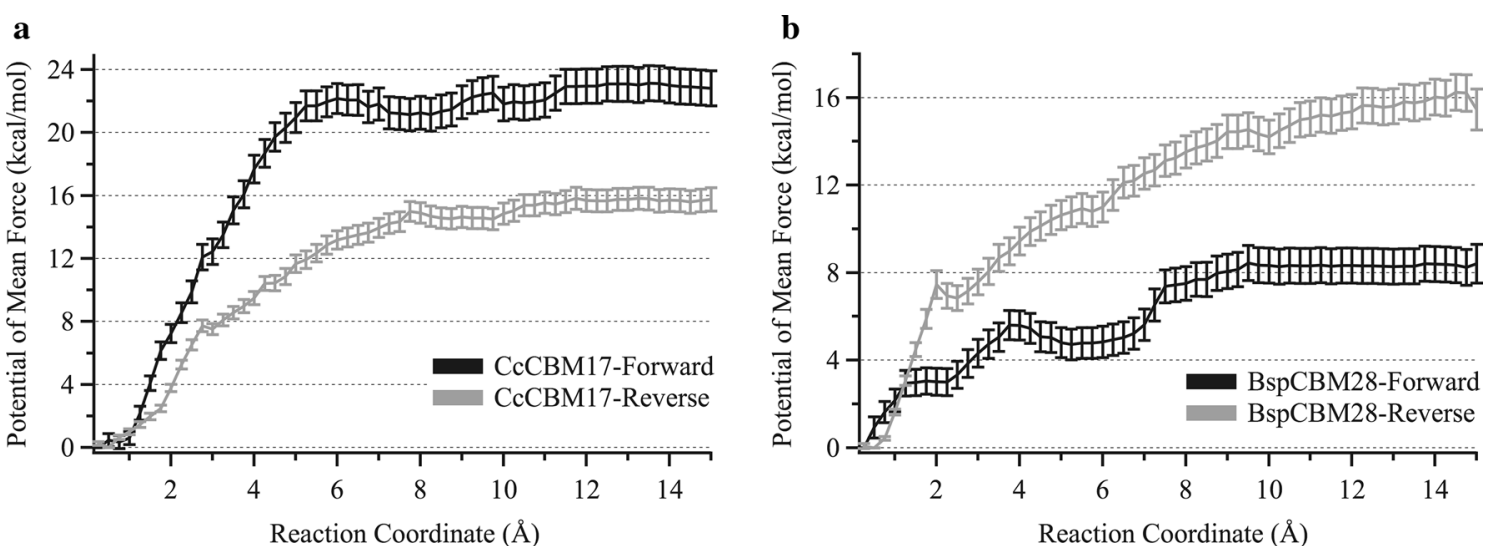

Fig. 9 Potential of mean force (PMF) in uncoupling a CCCBM17 and $\mathbf{b}$ BspCBM28 from non-crystalline cellulose. Umbrella sampling MD was conducted over 30 0.5-Å windows using the projection of the distance vector on the $z$-axis as the reaction coordinate

We used umbrella sampling MD to disassociate the CBM from non-crystalline cellulose, pulling the CBMs away from the substrate perpendicularly. Sampling simulations were started from equilibrated 100-ns MD simulation snapshots of each CBM-non-crystalline cellulose complex. For all four cases, the corresponding PMFs indicate binding affinities are higher for non-crystalline cellulose than for oligomeric ligands in respective CBMs (Fig. 9); this result aligns with our hypothesis that the higher affinity binding sites described in experimental binding studies corresponds to CBM-non-crystalline cellulose binding and lower affinity binding sites correspond to CBM binding in oligomeric or highly decrystallized regions.

The PMF provides both a binding free energy and a quantitative view of the CBM dissociation process from a non-crystalline substrate (Fig. 9). The free energy of binding non-crystalline cellulose is determined from the difference between the free energy at the beginning $(0$ $\AA$ ) and end $(15 \AA)$ of the reaction coordinate. For both CcCBM17 and BspCBM28, the orientation of the CBM relative to the surface affects binding affinity, favoring the forward orientation in CBM17 and the reverse orientation with CBM28. Additionally, there is a significant difference in affinity between the two high-affinity orientations of each CBM family; CcCBM17-F binds with the highest affinity, $23.0 \pm 1.1 \mathrm{kcal} / \mathrm{mol}$, and BspCBM28$\mathrm{R}$ binds with an affinity equivalent to $15.9 \pm 0.8 \mathrm{kcal} /$ mol. Combined with the knowledge that these two CBM families do not competitively bind non-crystalline cellulose [13], our results suggest that CBMs from these two families are capable of recognizing cellulose binding sites based on binding orientations relative to the substrate. The difference between the affinity of CBM17 and CBM28 for non-crystalline cellulose may be correlated to the qualitative difference in the surface interactions that contribute to the affinity as well as fortuitous compatibility of CBM17s than CBM28s with proposed noncrystalline cellulose model. Decrystallized edge chain morphology could be one of the other cases of noncrystalline cellulose that are preferred by CBM28s over CBM17s because of the differences in the general surface topology around binding site of oligomers.

The model non-crystalline substrate simulated in this study represents a subset of cellulose morphologies that are very close to crystalline substrate, and the calculated free energies correspond to association constants as high as $10^{12} \mathrm{~mol}^{-1}$, which are not detectable by experimental methods such as ITC. The reported high-affinity cellulose binding sites for $C c \mathrm{CBM} 17$ and $B s p \mathrm{CBM} 28$ on regenerated cellulose, from ITC, were $-8.41 \pm 0.32$ and $-8.28 \pm 0.35 \mathrm{kcal} / \mathrm{mol}$, respectively [13] and while these values are much lower than those calculated from PMFs, it is plausible that the experimental affinities correspond to a range of other cellulose morphologies more amorphous in nature than the model non-crystalline substrate. Nevertheless, taken qualitatively together with the calculated and experimental values of cellopentaose binding to $C c$ CBM17 and BspCBM28, our results offer promising evidence that high and low affinity non-crystalline cellulose binding sites correspond to the degree of substrate crystallinity. In other words, these family 17 and 28 CBMs appear to bind cellulose with a higher degree of crystallinity with greater affinity than small, oligomeric substrates.

Finally, dissociation appears to occur in two separate events along the PMF profile (Fig. 9), with an initial exertion of work to decouple the CBM from the substrate surface and a final extrication of the polymeric chain from the CBM binding groove. The CBM bound with 
non-crystalline cellulose must initially overcome the strong electrostatic interactions and hydrogen bonds formed between the CBM protein surface and the cellulose surface. After the exterior of the CBM was free of the cellulose surface, the final amount of work required to dissociate the CBM was associated with overcoming both van der Waals interactions between with the aromatic residues and pyranose rings and several hydrogen bonds formed with the substrate along the length of the groove. Combined with our MD simulation results above, the increase in affinity observed in binding CcCBM17 and $B s p$ CBM28 with non-crystalline cellulose appears to be directly related to the additional protein-carbohydrate interactions mediated by residues exterior to the CBM binding groove.

\section{Conclusions}

Through detailed analysis of protein-carbohydrate interactions, such as hydrogen bonding, and binding affinities for two different binding platforms observed within the same type of CBM, we found that binding site architecture appears to impact $\mathrm{CBM}$ functionality in recognizing carbohydrate substrates. Comparison of the twisted platforms in two different CBM families, 17 and 28, showed similarity in oligomeric ligand binding dynamics and established rationale towards possible extended binding sites. We have also addressed the questions raised by Boraston et al. in regards to mechanisms of Type B CBM-non-crystalline cellulose binding, expanding upon experimental observations identifying enthalpic interactions as dominant in non-crystalline substrate recognition by $C c$ CBM 17 and $B s p$ CBM28 [35]. Specifically, we identified individual contributions to thermodynamic parameters, revealing that the gain in enthalpy in binding non-crystalline cellulose over oligomers results from direct contact of the CBM exterior with the cellulose substrate. We also provided insight into how family 17 and 28 CBMs uncompetitively bind non-crystalline cellulose, despite having very similar binding specificities and protein structure. The question of specifically assigning CBM-cellulose binding affinities to non-crystalline substrate binding sites remains, hinging on future experimental efforts to structurally characterize non-crystalline cellulose of increasingly amorphous nature. This study also provides the basis for our future investigations of glycoside hydrolases linked with tandem CBMs, as the two family 4 CBMs (CfCBM4-1 and CfCBM4-2) and the two Bacillus sp. 1139 family 17 and 28 CBMs (BspCBM17 and $B s p C B M 28)$ are natural tandem constructs appended to $\beta$-1,4-endoglucanases. We anticipate the results toward understanding Type B CBM oligomeric and noncrystalline recognition mechanisms will advance our understanding of how protein-protein interactions and inter-module networking determines additive or cooperative binding in tandem systems and why organisms secret multi-modular enzymes with seemingly redundant CBM domains.

\section{Methods \\ Modeling protein-carbohydrate complexes}

Explicitly solvated models of each CBM were developed from Protein Data Bank (PDB) structures or via homology modeling. CfCBM4-1 and CfCBM4-2 models, in the apo and cellopentaose-bound states, were previously constructed [25]. CcCBM17 was constructed from the 1J84 PDB structure, which features cellotetraose bound in the groove [24]. Similarly, BspCBM28 was constructed from the 1 UWW PDB structure, having no bound ligand [36], and CjCBM28 was constructed from the 3ACI PDB structure, featuring cellopentaose [27]. With no available crystal structure for BspCBM17, we used homology modeling, with $C c \mathrm{CBM} 17$ as a template, to build the protein model [37, 38]; the two proteins are quite similar, having $55 \%$ sequence similarity and $70 \%$ structural similarity. For comparative purposes, we modeled the CBMbound cello-oligomer as cellopentaose; an additional $\beta$-D-glucose residue was constructed near the end of the $C c \mathrm{CBM} 17$ groove, and the cellopentaose ligand was docked with BspCBM17 and BspCBM28 by structural alignment with their homologous family member using the Dali pairwise alignment tool [39]. These four systems represent the oligomer-bound CBMs exhibiting the structural orientation, CcCBM17-RE, BspCBM17-RE, BspCBM28-NRE, and CjCBM28-NRE (Fig. 2a).

To investigate the bi-directional binding phenomenon in family 17 and 28 CBMs (Fig. 2b), we rotated the ligand from the structural orientation longitudinally along the ligand, as described for CfCBM4-1 and CfCBM4-2 [25]. Cellopentaose was docked in the opposite direction of that captured in the crystal structures by assuming the mean position of the pyranose ring heavy atoms must reside in approximately the same position regardless of direction. The approximate symmetry of the pyranose chair conformation enables this by merely exchanging the ring atom coordinates. CHARMM internal coordinate data was then used to establish the coordinates of the remaining sidechain atoms [40-42]. Extensive stepwise minimization of the ligand and the protein system was conducted before and after solvation to alleviate any deformation or bad contacts. These four systems, representing the "opposite" orientation, have been named CcCBM17-NRE, BspCBM17-NRE, BspCBM28-RE, and CjCBM28-RE for reference here.

We hypothesize high-affinity CBM-binding occurs when the CBM associates with amorphous or noncrystalline cellulose via partially decrystallized 
oligomeric chains decorating the top layers of degraded cellulose microfibrils (i.e., whiskers). Here, the partially decrystallized microfibril model used to represent amorphous/non-crystalline cellulose was adapted from the three-layer cellulose $\mathrm{I} \beta$ model used in previous cellulose decrystallization studies [43, 44]. The five-pyranose-long decrystallized segment was aligned with the cellopentaose from the equilibrated oligomeric systems described above using PyMOL (Additional file 1: Fig. S1). We docked two CBMs, a representative from both families 17 and 28 selected based on the availability of experimental affinity data for later comparison, in both ligand orientations such that we explore both possible interactions between these CBMs and non-crystalline cellulose. When aligned with each other or with $C f C B M 4-1-R E$ (Additional file 1: Fig. S2), CcCBM17 and BspCBM28, as obtained from crystal structures, appear to bind their cello-oligomeric ligands in opposite orientations, relative to the directionality of the core $\beta$-sheets. Assuming the structural orientations represent thermodynamically preferred recognition modes, we docked the $C c \mathrm{CBM} 17$ on the cellulose reducing end and $B s p C B M 28$ on the cellulose non-reducing end and refer to them as $C c \mathrm{CBM} 17-\mathrm{F}$ and BspCBM28-F (i.e., 'forward binding mode'). A second set of systems were prepared with the CBMs in the 'reverse binding mode,' exploring both bi-directional binding and additional CBM-substrate recognition mechanisms. These 'reverse' systems are referred as CcCBM17-R and BspCBM28-R (Fig. 2c). System construction was followed by extensive minimization and $1 \mathrm{~ns}$ of NPT equilibration to ensure the stability of the modeled proteincarbohydrate interaction and reduce solvation effects. During heating, equilibration, and production MD, the lower layer of the cellulose microfibril was restrained by applying harmonic restraints to the pyranose ring atoms; the CBMs and all other atoms of the systems were free of restraints. Protein alignment and ligand docking by alignment was carried out using PyMOL [45] and Dali pairwise comparison version 3.1 [39].

\section{MD simulation parameters and protocols}

The CHARMM36 force-field with CMAP corrections was used to model all proteins [46, 47], and carbohydrates were modeled with the CHARMM36 carbohydrate force-field [40-42]. Water molecules were represented by the modified TIP3P force-field $[48,49]$. Ions were modeled based on the force-field by Beglov and Roux [50]. Simulation setup and execution followed a procedure beginning with structure setup in CHARMM, vacuum energy minimization, explicit water solvation and charge neutralization with sodium ions, and extensive energy minimization [51]. The energy-minimized systems were then heated from 100 to $300 \mathrm{~K}$ over $20 \mathrm{ps}$ and then simulated in the NPT ensemble for 500 ps to equilibrate the solution density (except where noted above). After equilibration, each apo CBM and CBM-cellopentaose system was simulated for $250 \mathrm{~ns}$, and the CBM-microfibril systems were simulated in duplicate for $100 \mathrm{~ns}$ in the $N V T$ ensemble using NAMD [52]. Additional parameter and protocol details have been provided in Additional file 1 .

\section{Free energy calculations}

We calculated the absolute free energies of binding cellopentaose to CBMs for all three families using an enhanced sampling free energy method, FEP/ $\lambda$-REMD. $\mathrm{FEP} / \lambda$-REMD is an enhanced sampling free energy methodology developed by Jiang, Hodoscek, and Roux [53], which we have previously implemented for protein-carbohydrate systems obtaining good agreement with experimental data $[25,54,55]$. For two different systems, the CBM-cellopentaose complex in solvent and solvated cellopentaose, the non-bonded interactions of cellopentaose with the rest of the system were systematically turned off to obtain the change in free energy. This free energy calculation protocol was implemented using a dedicated module for replica exchange in NAMD [52]. The noncovalent interaction between the CBM and cellopentaose was distributed into repulsive, dispersive, electrostatic, and restraining components over 128 replicas. All replicas were simulated simultaneously for $0.1-\mathrm{ns}$, and more than 20 such $0.1-n$ consecutive windows were used to get 2-ns of sampling to ensure the convergence. The total change in free energy of binding was then calculated as the aggregate of $\Delta \mathrm{G}_{\text {repu }}, \Delta \mathrm{G}_{\text {disp }}, \Delta \mathrm{G}_{\text {elec }}$, and $\Delta \mathrm{G}_{\mathrm{rstr}}$. The difference between the free energy of 'disappearing' cellopentaose from the CBM groove into vacuum and the solvation free energy of cellopentaose gives the absolute free energy of binding a solvated ligand to a solvated protein. Convergence of the free energy values was determined by Multistate Bennett Acceptance Ratio (MBAR) analysis method [56] and can depend on whether the model was prepared from crystal structure or homology model. Free energy calculations using models implementing ligand docking or homology modeling included additional restraining forces to improve convergence. For direct comparison, the FEP/ $\lambda$-REMD calculations conducted here comply with the specifications outlined in our earlier study of family 4 CBMs [25]; accordingly, all methodological details are identical.

Umbrella sampling MD was used to determine the potential of mean force (PMF) of decoupling the CBM 
from the model non-crystalline surface into the solvent, from which we can estimate the free energy of binding. The distance between the projection of the center of mass of the CBM and the projection of center of mass of the lower layer of the cellulose microfibril on the $z$-axis served as the reaction coordinate. This distance was gradually increased by $15 \AA$ in $0.5 \AA$ increments, or 30 windows, until the non-bonded interaction between the protein and substrate no longer existed. The biasing force along the reaction coordinate was applied using collective variables during the 10-ns MD simulation of each window in NAMD [52]. To enable strictly perpendicular movement of the CBM relative to the microfibril surface, the distance between the same pair of projections on the $x$ - and $y$-axes was restrained as a constant. The harmonic restraint on the ring atoms of the lower layer of the microfibril was maintained throughout sampling. A force constant of $10 \mathrm{kcal} / \mathrm{mol}$ was used to maintain the collective variables to their specified values. In CcCBM17-F, the pyranose ring immediately prior to the decrystallized chain was harmonically restrained to the cellulose surface preventing further decrystallization as the CBM was pulled away. The last $5 \mathrm{~ns}$ of data was used in construction of the potential of mean force, discarding the first $5 \mathrm{~ns}$ as equilibration data. The reaction coordinates were normalized to represent the change in distance (i.e., $0 \AA$ to $15 \AA$ ). The calculation of potential of mean force profile and error analysis was performed using MBAR [56].

\section{Additional file}

Additional file 1. Details of molecular dynamics (MD) simulation methods and additional supporting figures and tables.

\section{Abbreviations}

CBMs: carbohydrate binding modules; MD: molecular dynamics; FEP/A-REMD: free energy perturbation with Hamiltonian replica exchange molecular dynamics; RMSF: root mean square fluctuation; SASA: solvent accessible surface area; ITC: isothermal titration calorimetry; PMF: potential of mean force; PDB: Protein Data Bank; MBAR: multistate Bennett acceptance ratio.

\section{Authors' contributions}

AAK participated in the design of the study, built molecular models, carried out molecular dynamics and free energy studies, performed the analysis and wrote the manuscript. CMP conceived, designed and coordinated the study, and drafted the manuscript. Both authors read and approved the final manuscript.

\section{Acknowledgements}

We acknowledge the computational time for this research provided by the Extreme Science and Engineering Discovery Environment (XSEDE) [57], which is supported by National Science Foundation Grant Number ACl-1548562. The work was conducted on the Pittsburgh Supercomputing Center Bridges cluster. Additional computational resources for testing and data analysis were made available by the Center for Computational Sciences DLX cluster at the University of Kentucky.

\section{Competing interests}

The authors declare that they have no competing interests.

\section{Availability of data and materials}

All data generated or analyzed during this study are included in this published article and its additional file.

\section{Consent for publication}

Not applicable.

\section{Ethics approval and consent to participate}

Not applicable.

\section{Funding}

Financial support was provided by the National Science Foundation (CHE1404849 to CMP). This material is also based upon work supported by (while CMP is serving at) the NSF. Any opinion, findings, and conclusions or recommendations expressed in this material are those of the authors and do not necessarily reflect the views of the NSF.

\section{Publisher's Note}

Springer Nature remains neutral with regard to jurisdictional claims in published maps and institutional affiliations.

Received: 28 July 2018 Accepted: 22 November 2018

Published online: 30 November 2018

\section{References}

1. Himmel ME, Ding SY, Johnson DK, Adney WS, Nimlos MR, Brady JW, Foust TD. Biomass recalcitrance: engineering plants and enzymes for biofuels production. Science. 2007;315:804-7.

2. Sun Y, Cheng JY. Hydrolysis of lignocellulosic materials for ethanol production: a review. Bioresourc Technol. 2002;83:1-11.

3. Boraston AB, Bolam DN, Gilbert HJ, Davies GJ. Carbohydrate-binding modules: fine-tuning polysaccharide recognition. Biochem J. 2004;382:769-81.

4. Shoseyov O, Shani Z, Levy I. Carbohydrate binding modules: biochemical properties and novel applications. Microbiol Mol Biol Rev. 2006;70:283-+

5. Linder $M$, Teeri TT. The roles and function of cellulose-binding domains. J Biotechnol. 1997;57:15-28.

6. Bolam DN, Ciruela A, McQueen-Mason S, Simpson P, Williamson MP, Rixon JE, Boraston A, Hazlewood GP, Gilbert HJ. Pseudomonas cellulose-binding domains mediate their effects by increasing enzyme substrate proximity. Biochem J. 1998:331:775-81.

7. Srisodsuk M, Lehtio J, Linder M, MargollesClark E, Reinikainen T, Teeri TT. Trichoderma reesei cellobiohydrolase I with an endoglucanase cellulosebinding domain: action on bacterial microcrystalline cellulose. J Biotechnol. 1997;57:49-57.

8. Tomme P, Boraston A, McLean B, Kormos J, Creagh AL, Sturch K, Gilkes NR, Haynes CA, Warren RAJ, Kilburn DG. Characterization and affinity applications of cellulose-binding domains. J Chromatogr B. 1998;715:283-96.

9. Boraston AB, Creagh AL, Alam MM, Kormos JM, Tomme P, Haynes CA, Warren RAJ, Kilburn DG. Binding specificity and thermodynamics of a family 9 carbohydrate-binding module from Thermotoga maritima xylanase 10A. Biochemistry. 2001:40:6240-7.

10. Notenboom V, Boraston AB, Kilburn DG, Rose DR. Crystal structures of the family 9 carbohydrate-binding module from Thermotoga maritima xylanase 10A in native and ligand-bound forms. Biochemistry. 2001;40:6248-56.

11. Johnson PE, Brun E, Mackenzie LF, Withers SG, Mclntosh LP. The cellulosebinding domains from Cellulomonas fimi beta-1, 4-glucanase CenC bind nitroxide spin-labeled cellooligosaccharides in multiple orientations. J Mol Biol. 1999;287:609-25.

12. Carrard G, Koivula A, Soderlund H, Beguin P. Cellulose-binding domains promote hydrolysis of different sites on crystalline cellulose. Proc Natl Acad Sci USA. 2000;97:10342-7. 
13. Boraston AB, Kwan E, Chiu P, Warren RAJ, Kilburn DG. Recognition and hydrolysis of noncrystalline cellulose. J Biol Chem. 2003;278:6120-7.

14. Gilbert HJ, Knox JP, Boraston AB. Advances in understanding the molecular basis of plant cell wall polysaccharide recognition by carbohydratebinding modules. Curr Opin Struct Biol. 2013;23:669-77.

15. Tormo J, Lamed R, Chirino AJ, Morag E, Bayer EA, Shoham Y, Steitz TA. Crystal structure of a bacterial family-III cellulose-binding domain: a general mechanism for attachment to cellulose. EMBO J. 1996:15:5739-51.

16. McLean BW, Bray MR, Boraston AB, Gilkes NR, Haynes CA, Kilburn DG. Analysis of binding of the family 2 a carbohydrate-binding module from Cellulomonas fimi xylanase 10A to cellulose: specificity and identification of functionally important amino acid residues. Protein Eng. 2000;13:801-9.

17. McLean BW, Boraston AB, Brouwer D, Sanaie N, Fyfe CA, Warren RAJ, Kilburn DG, Haynes CA. Carbohydrate-binding modules recognize fine substructures of cellulose. J Biol Chem. 2002;277:50245-54.

18. Ciolacu D, Ciolacu F, Popa VI. Amorphous cellulose-structure and characterization. Cellul Chem Technol. 2011;45:13-21.

19. Teeri TT, Koivula A, Linder M, Wohlfahrt G, Divne C, Jones TA. Trichoderma reesei cellobiohydrolases: why so efficient on crystalline cellulose? Biochem Soc Trans. 1998;26:173-8.

20. Tomme P, Driver DP, Amandoron EA, Miller RC, Antony R, Warren J, Kilburn DG. Comparison of a fungal (Family-l) and bacterial (Family-li) cellulosebinding domain. J Bacteriol. 1995;177:4356-63.

21. Boraston AB, Ghaffari M, Warren RAJ, Kilburn DG. Identification and glucan-binding properties of a new carbohydrate-binding module family. Biochem J. 2002;361:35-40.

22. Boraston AB, Chiu P, Warren RAJ, Kilburn DG. Specificity and affinity of substrate binding by a family 17 carbohydrate-binding module from Clostridium cellulovorans cellulase 5A. Biochemistry. 2000:39:11129-36.

23. Tomme P, Creagh AL, Kilburn DG, Haynes CA. Interaction of polysaccharides with the $\mathrm{N}$-terminal cellulose-binding domain of Cellulomonas fimi CenC.1. Binding specificity and calorimetric analysis. Biochemistry. 1996;35:13885-94.

24. Notenboom V, Boraston AB, Chiu P, Freelove ACJ, Kilburn DG, Rose DR. Recognition of cello-oligosaccharides by a family 17 carbohydrate-binding module: an X-ray crystallographic, thermodynamic and mutagenic study. J Mol Biol. 2001;314:797-806.

25. Kognole AA, Payne CM. Cello-oligomer-binding dynamics and directionality in family 4 carbohydrate-binding modules. Glycobiology. 2015:25:1100-11.

26. Boraston AB, Nurizzo D, Notenboom V, Ducros V, Rose DR, Kilburn DG, Davies GJ. Differential oligosaccharide recognition by evolutionarily-related $\beta-1,4$ and $\beta-1,3$ glucan-binding modules. J Mol Biol. 2002;319:1143-56.

27. Tsukimoto K, Takada R, Araki Y, Suzuki K, Karita S, Wakagi T, Shoun H, Watanabe T, Fushinobu S. Recognition of cellooligosaccharides by a family 28 carbohydrate-binding module. FEBS Lett. 2010;584:1205-11.

28. Cao RY, Jin YD, Xu DG. Recognition of cello-oligosaccharides by CBM17 from Clostridium cellulovorans: molecular dynamics simulation. J Phys Chem B. 2012;116:6087-96.

29. Araki Y, Karita S, Tanaka A, Kondo M, Goto M. Characterization of family 17 and family 28 carbohydrate-binding modules from Clostridium josui Cel5A. Biosci Biotechnol Biochem. 2009;73:1028-32.

30. Bronowska AK. Thermodynamics of ligand protein interactions implications for molecular design. New York: InTech; 2011.

31. Emsley J. Very strong hydrogen bonding. Chem Soc Rev. 1980;9:91-124.

32. Cockburn D, Svensson B. Surface binding sites in carbohydrate active enzymes: an emerging picture of structural and functional diversity. Carbohydr Chem. 2013;39:204-21

33. Araki Y, Karita S, Tsuchiya T, Kondo M, Goto M. Family 17 and 28 carbohydrate-binding modules discriminated different cell-wall sites in sweet potato roots. Biosci Biotechnol Biochem. 2010;74:802-5.

34. Payne CM, Resch MG, Chen LQ, Crowley MF, Himmel ME, Taylor LE, Sandgren M, Stahlberg J, Stals I, Tan ZP, Beckham GT. Glycosylated linkers in multimodular lignocellulose-degrading enzymes dynamically bind to cellulose. Proc Natl Acad Sci USA. 2013;110:14646-51.

35. Boraston AB. The interaction of carbohydrate-binding modules with insoluble non-crystalline cellulose is enthalpically driven. Biochem 2005:385:479-84.
36. Jamal S, Nurizzo D, Boraston AB, Davies GJ. X-Ray crystal structure of a non-crystalline cellulose-specific carbohydrate-binding module: CBM28. J Mol Biol. 2004:339:253-8.

37. Biasini M, Bienert S, Waterhouse A, Arnold K, Studer G, Schmidt T, Kiefer F, Cassarino TG, Bertoni M, Bordoli L, Schwede T. SWISS-MODEL: modelling protein tertiary and quaternary structure using evolutionary information. Nucleic Acids Res. 2014:42:W252-8.

38. Arnold K, Bordoli L, Kopp J, Schwede T. The SWISS-MODEL workspace: a web-based environment for protein structure homology modelling. Bioinformatics. 2006;22:195-201.

39. Hasegawa H, Holm L. Advances and pitfalls of protein structural alignment. Curr Opin Struct Biol. 2009;19:341-8.

40. Guvench O, Greene SN, Kamath G, Brady JW, Venable RM, Pastor RW, MacKerell AD. Additive empirical force field for hexopyranose monosaccharides. J Comput Chem. 2008;29:2543-64.

41. Guvench O, Hatcher E, Venable RM, Pastor RW, MacKerell AD. CHARMM additive all-atom force field for glycosidic linkages between hexopyranoses. J Chem Theory Comput. 2009;5:2353-70.

42. Guvench O, Mallajosyula SS, Raman EP, Hatcher E, Vanommeslaeghe K, Foster TJ, Jamison FW, Mackerell AD. CHARMM additive all-atom force field for carbohydrate derivatives and its utility in polysaccharide and carbohydrate-protein modeling. J Chem Theory Comput. 2011;7:3162-80.

43. Beckham GT, Matthews JF, Peters B, Bomble YJ, Himmel ME, Crowley MF. Molecular-level origins of biomass recalcitrance: decrystallization free energies for four common cellulose polymorphs. J Phys Chem B. 2011;115:4118-27.

44. Payne CM, Himmel ME, Crowley MF, Beckham GT. Decrystallization of oligosaccharides from the cellulose I beta surface with molecular simulation. J Phys Chem Lett. 2011;2:1546-50.

45. Schrodinger, LLC. The PyMOL molecular graphics system, version $1.1 \mathrm{r} 1$. New York: Schrodinger, LLC; 2010.

46. MacKerell AD, Bashford D, Bellott M, Dunbrack RL, Evanseck JD, Field MJ, Fischer S, Gao J, Guo H, Ha S, et al. All-atom empirical potential for molecular modeling and dynamics studies of proteins. J Phys Chem B. 1998;102:3586-616.

47. MacKerell AD, Feig M, Brooks CL. Extending the treatment of backbone energetics in protein force fields: limitations of gas-phase quantum mechanics in reproducing protein conformational distributions in molecular dynamics simulations. J Comput Chem. 2004;25:1400-15.

48. Jorgensen WL, Chandrasekhar J, Madura JD, Impey RW, Klein ML. Comparison of simple potential functions for simulating liquid water. J Chem Phys. 1983:79:926-35.

49. Durell SR, Brooks BR, Bennaim A. Solvent-induced forces between 2 hydrophilic groups. J Phys Chem. 1994;98:2198-202.

50. Beglov D, Roux B. Finite representation of an infinite bulk system: solvent boundary potential for computer simulations. J Chem Phys. 1994;100:9050-63.

51. Brooks BR, Brooks CL, Mackerell AD, Nilsson L, Petrella RJ, Roux B, Won Y, Archontis G, Bartels C, Boresch S, et al. CHARMM: the biomolecular simulation program. J Comput Chem. 2009;30:1545-614.

52. Phillips JC, Braun R, Wang W, Gumbart J, Tajkhorshid E, Villa E, Chipot C, Skeel RD, Kale L, Schulten K. Scalable molecular dynamics with NAMD. J Comput Chem. 2005:26:1781-802.

53. Jiang W, Hodoscek M, Roux B. Computation of absolute hydration and binding free energy with free energy perturbation distributed replicaexchange molecular dynamics. J Chem Theory Comput. 2009;5:2583-8.

54. Payne CM, Jiang W, Shirts MR, Himmel ME, Crowley MF, Beckham GT. Glycoside hydrolase processivity is directly related to oligosaccharide binding free energy. J Am Chem Soc. 2013;135:18831-9.

55. Hamre AG, Jana S, Holen MM, Mathiesen G, Valjamae P, Payne CM, Sorlie M. Thermodynamic relationships with processivity in Serratia marcescens family 18 chitinases. J Phys Chem B. 2015;119:9601-13.

56. Shirts MR, Chodera JD. Statistically optimal analysis of samples from multiple equilibrium states. J Chem Phys. 2008;129:1-10.

57. Towns J, Cockerill T, Dahan M, Foster I, Gaither K, Grimshaw A, Hazlewood V, Lathrop S, Lifka D, Peterson GD, et al. XSEDE: accelerating scientific discovery. Comput Sci Eng. 2014;16:62-74.

58. Brun E, Johnson PE, Creagh AL, Tomme P, Webster P, Haynes CA, McIntosh LP. Structure and binding specificity of the second N-terminal cellulosebinding domain from Cellulomonas fimi endoglucanase C. Biochemistry. 2000:39:2445-58. 